\title{
Belphégor
}

\section{Ann Miller \& Bart Beaty (eds), The French Comics Theory Reader}

\section{Chris Reyns-Chikuma}

\section{(2) OpenEdition}

1 Journals

\section{Édition électronique}

URL : http://journals.openedition.org/belphegor/556

DOI : 10.4000/belphegor.556

ISSN : 1499-7185

Éditeur

LPCM

\section{Référence électronique}

Chris Reyns-Chikuma, "Ann Miller \& Bart Beaty (eds), The French Comics Theory Reader », Belphégor [En ligne], 13-1 | 2015, mis en ligne le 09 mai 2015, consulté le 22 septembre 2020. URL : http:// journals.openedition.org/belphegor/556 ; DOI : https://doi.org/10.4000/belphegor.556

Ce document a été généré automatiquement le 22 septembre 2020.

\section{(c) (i) (9)}

Belphégor est mis à disposition selon les termes de la Licence Creative Commons Attribution - Pas d'Utilisation Commerciale - Pas de Modification 4.0 International. 


\section{Ann Miller \& Bart Beaty (eds), The French Comics Theory Reader}

Chris Reyns-Chikuma

\section{RÉFÉRENCE}

Ann Miller \& Bart Beaty (eds), The French Comics Theory Reader, Leuven : Leuven University Press LUP, 2014.ISBN : 9789058679888 
1 Ceci est le premier volume d'une collection toute nouvelle (2014), intitulée "Studies in European Comics and Graphics Novels" publiée par une maison d'édition elle aussi relativement nouvelle (1971). Le deuxième volume vient aussi de sortir cette année et est consacré au grand Sfar (voir mon deuxième compte rendu dans ce numéro).

2 Le fait de parler de "European Comics ", c'est-à-dire de bande dessinée européenne, n'est pas nouveau en Europe. Ainsi dès 2000, le belge Thierry Groensteen avait intitulé l'un de ses livres Maîtres de la bande dessinée européenne. Mais cette appellation n'est pas encore commune dans le monde anglophone qui

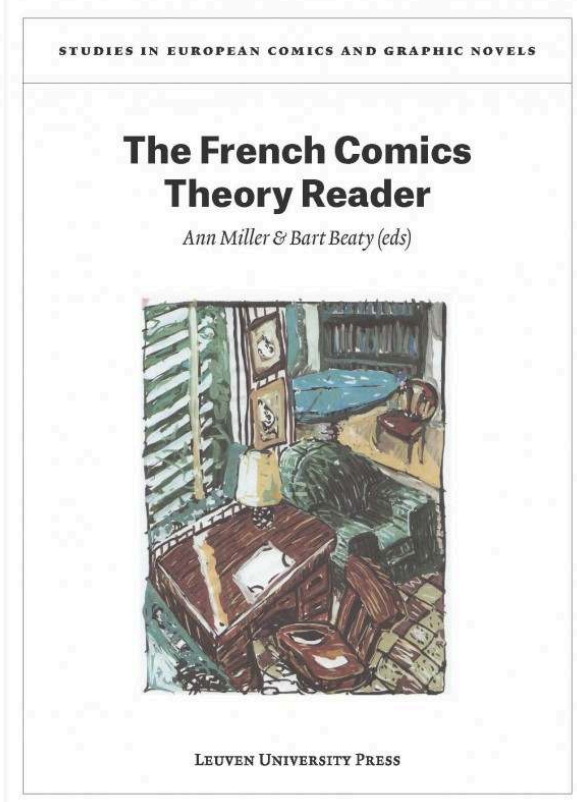
parle encore souvent de tradition nationale ou dans le cas de la bande dessinée en français, de la tradition franco-belge, même si Beaty avait intitulé son livre en 2007, Unpopular Culture: Transforming the European Comic Book in the 1990s. Pour les auteurs de cet ouvrage comme pour les éditeurs de cette collection, c'est aussi une manière de relativiser la place de la bande dessinée française ou franco-belge face aux nombreuses autres traditions nationales ou régionales, anciennes ou plus récentes, en langue flamande (Leuven est une université flamande), allemande, italienne ...

3 Pour les lecteurs européens, le "reader" fait référence à un genre aujourd'hui typiquement anglo-saxon où il est très répandu comme manuel de lecture. Il pourrait être comparé en France à l'anthologie qui existe peu pour des textes critiques ou théoriques contemporains. Cette anthologie rassemble 25 textes écrits en français par des auteurs français, belges francophones et néerlandophones mais d'horizons assez différents : historiens, sociologues, critiques, théoriciens de la bande dessinée de trois générations. La première génération, les Lacassin, Fresnault-Deruelle, Ory, puis une seconde génération, les Groensteen, Peeters, Lefevre, et enfin ceux des années 1990-2010 avec les Baetens, Smolderen, et Morgan...

4 Comme les auteurs l'expliquent, sauf pour quelques allusions dans les introductions, l'anthologie ne contient aucun texte antérieur à 1970. La plupart de ces textes, qui font de deux à vingt pages, sont connus des spécialistes ou amoureux de la bande dessinée en français, mais pas nécessairement des chercheurs anglo-saxons souvent enfermés dans leur univers déjà si riche des "Superheroes » et des "graphic novels ». Et c'est évidemment le premier but des auteurs de leur faire connaitre ces textes dans une culture de plus en plus internationale, où Maus a indéniablement été influencé par la bande dessinée européenne, Satrapi est traduite dans 30 langues, et Watchmen et $V$ for Vendetta ont une influence considérable au-delà de leur Angleterre natale. Conséquemment, ces textes seront ainsi aussi disponibles pour des spécialistes des comics d'autres langues aux traditions longues et riches comme le manga japonais ou mangwa chinois qui ont plus de chances de parler/lire l'anglais que le français. 
Le livre est divisé en quatre sections. La première intitulée "Origines et définitions ", contient huit textes, dont le premier, de Glasser, porte sur l'origine récente du mot de bande dessinée. On compte également deux textes de Groensteen, un texte de Blanchard, un autre de Lacassin et deux textes de Sylvain Bouyer. La deuxième, "Approches formelles de la bande dessinée ", propose six textes dominés par la tradition sémiotique de Fresnault-Deruelle: textes de Sterckx, Samson, Groensteen, Baetens et Lefevre. La troisième, «la critique », offre six textes de noms connus comme Serres, Peeters, Tisseron, Morgan, Lecigne, et encore Fresnault-Deruelle. Enfin, la quatrième, "L'industrie de la bande dessinée ", contient cinq textes, dont ceux très célèbres de Boltanski et Ory, ceux moins connus mais tout aussi intéressants de Dejasse et Schwartz, et un extrait du pamphlet fameux de Christophe Menu.

Chaque partie est précédée d'une bonne introduction de quelques pages qui présente les articles ou extraits sélectionnés en un ou deux paragraphes. Chaque texte est aussi suivi de quelques notes, certaines venant du texte original, d'autres ajoutées par les auteurs-traducteurs («translator's note») pour éclaircir certains points trop spécifiques au monde francophone comme la note 1 sur l'influente agence de presse et de syndication française Opera Mundi créée par Paul Winckler en 1928 (p. 23). L'anthologie contient aussi une vingtaine d'illustrations.

7 Pour conclure, ce livre est un outil des plus utiles, édité par deux des plus grands spécialistes de la bande dessinée de la nouvelle génération anglophone. L'intérêt de leur perspective est double. D'une part, ils connaissent bien la tradition européenne ayant tous les deux publié plusieurs livres et plusieurs articles sur celle-ci, d'autre part, dans la mesure où ils ont aussi publié des études sur les autres traditions (anglaise/ britannique, canadienne et américaine), ils ont une distance culturelle et linguistique vis-à-vis de cette tradition européenne, chose qui n'est pas toujours le cas pour les critiques francophones. De plus, cette tradition anglo-saxonne, ancrée dans les Communication Studies pour Beaty et les Cultural Studies pour Miller est régulièrement citée ou alludée dans les introductions, incluant parfois la perspective des Colonial Studies et des Gender Studies, domaines qui commencent seulement à se développer dans les études de la bande dessinée en France et en Belgique si longtemps dominées par la sémiologie d'une part, et par l'histoire et la psychanalyse d'autre part.

\section{AUTEURS}

\section{CHRIS REYNS-CHIKUMA}

University of Alberta 